\title{
Oscillatory squeeze flow for the study of linear viscoelastic behavior
}

\author{
Wingstrand, Sara Lindeblad; Alvarez, Nicolas J.; Hassager, Ole; Dealy, John M.
}

Published in:

Journal of Rheology

Link to article, DOI:

$10.1122 / 1.4943984$

Publication date:

2016

Document Version

Publisher's PDF, also known as Version of record

Link back to DTU Orbit

Citation (APA):

Wingstrand, S. L., Alvarez, N. J., Hassager, O., \& Dealy, J. M. (2016). Oscillatory squeeze flow for the study of linear viscoelastic behavior. Journal of Rheology, 60(3), 407-418. https://doi.org/10.1122/1.4943984

\section{General rights}

Copyright and moral rights for the publications made accessible in the public portal are retained by the authors and/or other copyright owners and it is a condition of accessing publications that users recognise and abide by the legal requirements associated with these rights.

- Users may download and print one copy of any publication from the public portal for the purpose of private study or research.

- You may not further distribute the material or use it for any profit-making activity or commercial gain

- You may freely distribute the URL identifying the publication in the public portal

If you believe that this document breaches copyright please contact us providing details, and we will remove access to the work immediately and investigate your claim. 


\section{JOURNAL OF RHEOLOGY}

\section{Oscillatory squeeze flow for the study of linear viscoelastic behavior}

Sara L. Wingstrand, Nicolas J. Alvarez, Ole Hassager, and John M. Dealy

Citation: Journal of Rheology 60, 407 (2016); doi: 10.1122/1.4943984

View online: http://dx.doi.org/10.1122/1.4943984

View Table of Contents: http://scitation.aip.org/content/sor/journal/jor2/60/3?ver=pdfcov

Published by the The Society of Rheology

\section{Articles you may be interested in}

Large amplitude oscillatory microrheology

J. Rheol. 58, 1 (2014); 10.1122/1.4826939

Rheology of concentrated soft and hard-sphere suspensions

J. Rheol. 57, 1195 (2013); 10.1122/1.4808054

Effect of flow history on linear viscoelastic properties and the evolution of the structure of multiwalled carbon nanotube suspensions in an epoxy

J. Rheol. 55, 153 (2011); 10.1122/1.3523628

Oscillatory shear of suspensions of noncolloidal particles

J. Rheol. 50, 711 (2006); 10.1122/1.2234366

Model Analysis of Shear-Flow Behavior of Linear Low-Density Polyethylene (LLDPE) Using a Simple Integral Constitutive Equation

J. Rheol. 29, 726 (1985); 10.1122/1.549807

The World's Most Versatile Platform for Rheological Measurements
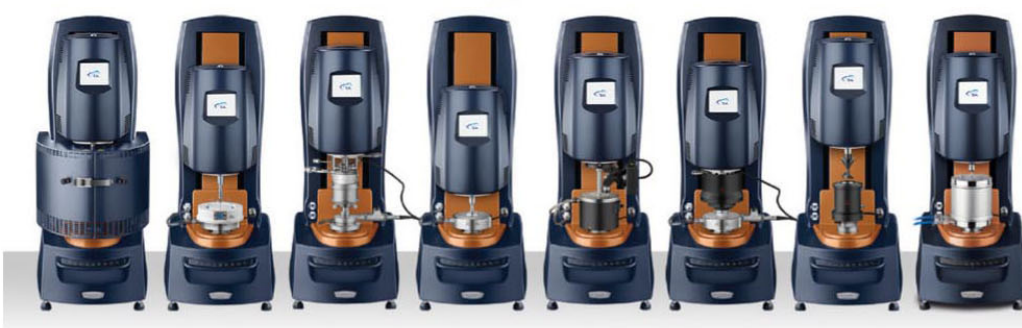

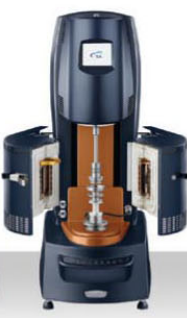

The Discovery

Hybrid Rheometer from

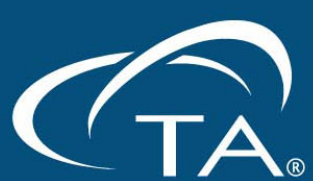

Instruments 


\title{
Oscillatory squeeze flow for the study of linear viscoelastic behavior
}

\author{
Sara L. Wingstrand \\ Department of Chemical and Biochemical Engineering, Technical University of Denmark, \\ DK-2800 Kgs Lyngby, Denmark
}

Nicolas J. Alvarez

Department of Chemical and Biological Engineering, Drexel University, Philadelphia, Pennsylvania 19104

Ole Hassager ${ }^{a)}$

Department of Chemical and Biochemical Engineering, Technical University of Denmark, DK-2800 Kgs Lyngby, Denmark

John M. Dealy

Department of Chemical Engineering, McGill University, Montreal, Quebec H3A 0C4, Canada

(Received 28 September 2015; final revision received 9 February 2016; published 28 March 2016)

\begin{abstract}
The squeezing of a sample between parallel plates has been used for many years to characterize the rheological behavior of soft, purely viscous materials, and in recent times, small-amplitude oscillatory squeezing has been proposed as a means to determine the linear viscoelastic properties of molten polymers and suspensions. The principal advantage of squeeze flow rheometer over rotational devices is the simplicity of the apparatus. It has no air bearing and is much less expensive and easier to use. Accuracy may be somewhat reduced, but for quality control purposes, it could be quite useful. It might also find application as the central component of a high-throughput rheometer for evaluating experimental materials. The deformation is not simple shear, but equations have been derived to show that the oscillatory compressive (normal) force that is measured can serve as a basis for calculating the storage and loss moduli. These theories as well as instruments that have been developed to generate the required deformation are described, and applications to a variety of materials are described. (C) 2016 The Society of Rheology. [http://dx.doi.org/10.1122/1.4943984]
\end{abstract}

\section{INTRODUCTION}

Engmann et al. [1] published a useful review of squeeze flow rheometry, emphasizing its use for fluids with yield stress but saying little about oscillatory squeeze flow. We begin with a brief review of the use of squeezing flows for the characterization of purely viscous (inelastic) materials. Squeezing flow is not a viscometric flow, and devices using this deformation for non-Newtonian fluids can be used only for qualitative purposes unless a constitutive model is available.

There are two methods of operation: constant area and constant volume. In a "squeeze film" device, the sample initially has the same size as the circular plates between which it is compressed so that the area of the sample under compression is constant. A constant normal force, $F$, is applied to the upper plate, most simply by means of a weight, and the decreasing plate spacing, $H(t)$, is measured. In a "parallel-plate plastometer," the sample is smaller than the plates so that the sample volume remains constant rather

\footnotetext{
a) Author to whom correspondence should be addressed; electronic mail: oh@kt.dtu.dk

than its compressed area. A device based on this concept is used in a standard test method for measuring an "apparent viscosity" of bitumen [2]. The constant area method is sometimes preferred, because if a smaller sample is used, its volume and thus its radius during testing must be precisely known. It is also desirable that it be centered in the gap to ensure symmetric loading.

Another mode of operation involves a constant speed rather than a constant normal force with the force monitored as a function of time, and there are thus four possible combinations of constant-volume/constant-radius and constant-speed/constant-force. Some devices also work at exponentially decaying speed to achieve a nominally constant rate of compression.

The modeling of nonoscillatory squeeze flow assumes that inertia can be neglected (very low Re) and that the velocity distribution has a certain form. Equations for the interpretation of data in terms of viscosity are described in detail by Bird et al. [3], and we present here only a brief summary. Considering first constant area flow, for a Newtonian fluid, the deformation is governed by the Stefan equation, shown as 


$$
F(t)=\frac{3 \pi R^{4} \eta(-d H / d t)}{2[H(t)]^{3}} .
$$

Here, $R$ is the radius of the sample and $\eta$ is the Newtonian viscosity. For a constant compressive (normal) force $F_{0}$ and initial condition $H(0)=H_{0}$, the gap spacing is given by

$$
\frac{1}{H^{2}}=\frac{1}{H_{0}^{2}}+\frac{4 F_{0} t}{3 \pi R_{0}^{4} \eta} .
$$

At very short times, inertia cannot be neglected, so if $\left(1 / H^{2}\right)$ is plotted versus time, the first points will not lie on a line, but if the data approach a line, the slope can be used to calculate the viscosity. If the deformation is sufficiently slow, this technique should provide a reasonable approximation of the zero-shear viscosity of a molten polymer.

For a constant volume flow, Dienes and Klemm [4] derived the following relationship for a Newtonian fluid, where $V$ is the volume of the sample:

$$
F=\frac{3 \eta V^{2}}{2 \pi H^{5}}\left(-\frac{d H}{d t}\right) .
$$

For a constant normal force $F_{0}$ and a constant volume, integration with the initial condition $H(0)=H_{0}$ gives

$$
\frac{1}{H^{4}}=\frac{1}{H_{0}^{4}}+\frac{8 \pi F_{0}}{3 \eta V^{2}} t
$$

Gent [5] reported that the assumptions regarding the velocity profile are only valid when $R_{0}>10 H_{0}(\Lambda<0.1)$.

Because the strain is not uniform throughout the flow, the interpretation of data for non-Newtonian fluids requires a viscosity model, and Engmann et al. [1] have compiled an extensive list of all the fluid models for which squeeze flow equations have been reported, including both inelastic and elastic materials and with no-slip, slip, and partial-slip boundary conditions. For example, for a power-law fluid with consistency index $m$ and power law parameter $n$, the Scott equation describes the flow with a constant force and volume

$$
F=\left(\frac{2 \pi m R^{n+3}}{n+3}\right)\left(\frac{2 n+1}{n}\right) \frac{(-d H / d t)^{n}}{H^{2 n+1}} .
$$

Gibson et al. [6] used squeezing flow to study the behavior of suspensions of planar fibers in polypropylene. Pham and Meinecke [7,8] employed squeezing flow at a constant plate speed rather than a constant normal force for the evaluation of commercial polymers. Shaw [9] used a squeeze-flow apparatus to deal with the difficult problem of characterizing the flow behavior of ultrahigh-density polyethylene, which has a very high viscosity. The driving pressure was maintained constant, and $H(t)$ was measured, Even at very low shear rates, this material is still pseudoplastic, but the Stefan equation was used to calculate an apparent viscosity that was related to molecular weight by an empirically established relationship. About $25 \mathrm{~min}$ were required for a measurement. Cua and Shaw [10] used a similar method to determine the low-shear rate viscosity of a polydimethylsiloxane.
Squeeze flow has also been used to measure the response of molten polymers to biaxial extension, a deformation that is difficult to generate in the laboratory. To address this problem, squeezing between lubricated plates (LSF) has been used to eliminate the shearing component of the flow field. The LSF method of biaxial stretching was first proposed by Chatraei et al. [11] and was later used by Nishioka et al. [12] and by Kompani and Venerus [13]. The latter authors replenished the lubricant continuously during a test to avoid the development of dry regions. They reviewed the history of the LSF technique and developed the basic equations for the interpretation of data in terms of the biaxial extensional viscosity. Guaderama-Medina et al. [14] compared results for a low-density polyethylene obtained by LSF with those obtained using a complex instrument in which a circular sheet of polymer is stretched by six pairs of rotary clamps [15]. It was found that the LSF data were not reliable at Hencky strains greater than one.

\section{THEORY OF OSCILLATORY SQUEEZE FLOW}

We turn now to small amplitude oscillatory flow as described by the theory of linear viscoelasticity. We begin by recalling the correspondence principle by Pipkin [16], which applies to general quasisteady deformations (i.e., no inertia). Pipkin demonstrated that it is possible to go from the solution to an equilibrium elasticity problem to a corresponding quasisteady linearly viscoelastic problem. In a similar development, Phan-Thien [17] and Field et al. [18] transformed the Stefan equation for Newtonian fluids to the corresponding functional relation for linear viscoelastic materials, again for deformations with negligible inertia by appropriate substitution of the constant viscosity by the complex viscosity. We make use of this relationship below [Eq. (46)].

We here describe how linear viscoelastic behavior can be measured by the use of squeeze flow, but in a form that is not necessarily restricted to negligible inertia.

Consider a fluid sandwiched between two plates as illustrated in Fig. 1. With the bottom plate located at an axial position $z=0$, the top plate position $(H)$ also signifies the height of the gap between the top and bottom plates. The top plate oscillates axially about a mean height $H_{0}$ to impose oscillatory squeezing described by

$$
H(t)=H_{0}(1+a \sin \omega t) .
$$

Here, $H_{0}$ is the top plate position at time $t=0, a H_{0}$ is the amplitude, and $\omega$ is frequency. The speed of the top plate $\dot{H}$ can thus be expressed as

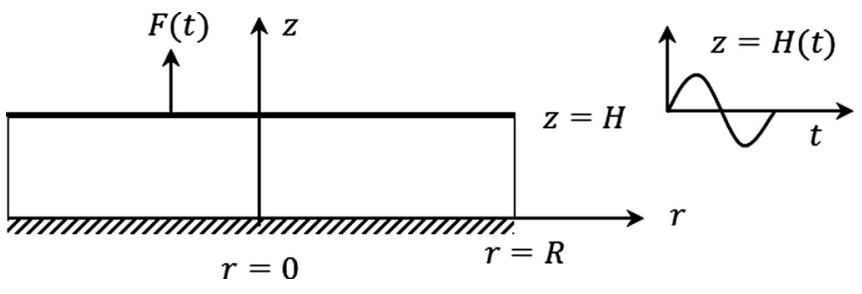

FIG. 1. Sketch of an oscillatory squeeze flow geometry. 


$$
\dot{H}=\frac{d H}{d t}=a H_{0} \omega \Re\left\{e^{i \omega t}\right\},
$$

where $\Re\{\}$ indicates the real part of the complex quantity inside the brackets.

As we are concerned here only with linear viscoelastic characterization, $a$ is assumed to be small. The permissible magnitude of $a$ depends on the aspect ratio $\Lambda \equiv H_{0} / R_{0}$. Konigsberg [19] has discussed the problem of defining a characteristic value of strain, but the value of the maximum strain, which occurs at the rim, can be evaluated, as shown in the following discussion, and this governs the limit for the applicability of the linear viscoelasticity (LVE) governing equations. By restricting the analysis to small deformations the following simplifications can be made:

(1) The convection term $(v \cdot \nabla v)$ in the equation of motion, where $v$ is velocity, can be neglected, as it is of order $O\left(a^{2}\right)[20] .^{1}$

(2) The governing equations of linear viscoelasticity may be used.

Based on these considerations $v$ and the pressure $p$ can be represented as follows:

$$
\begin{aligned}
& \boldsymbol{v}=\Re\left\{\overline{\boldsymbol{v}}(\boldsymbol{x}) e^{i \omega t}\right\}, \\
& p=\Re\left\{\bar{p}(\boldsymbol{x}) e^{i \omega t}\right\},
\end{aligned}
$$

where $\overline{\boldsymbol{v}}(\boldsymbol{x})$ and $\bar{p}(\boldsymbol{x})$ are complex functions that only vary spatially so that the extra stress tensor $\sigma$ can be written simply as

$$
\boldsymbol{\sigma}=\Re\left\{\eta^{*}\left[(\nabla \overline{\boldsymbol{v}})+\left(\nabla \overline{\boldsymbol{v}}^{\dagger}\right)\right] e^{i \omega t}\right\}
$$

It follows that the velocity field for flow of a linear viscoelastic fluid is described by the governing equations of a Newtonian fluid, i.e., Navier-Stokes equations, with viscosity replaced by the complex viscosity $\eta^{*}$. This is in accord with the correspondence principle of Pipkin [16] who showed a similar analogy to elasticity problems. Here, we use this technique to derive the velocity distribution for the oscillatory squeeze flow.

In setting up the equations of motion, we assume incompressibility and neglect flow in the angular direction $(\theta)$ so that $v_{\theta}=0$, and $\partial / \partial \theta=0$. Also, we introduce dimensionless complex variables $\hat{r}, \hat{z}, \hat{v}_{r}, \hat{v}_{z}$, and $\hat{p}$ as follows:

$$
\begin{gathered}
\hat{r}=r / R_{0}, \\
\hat{z}=z / H_{0}, \\
v_{r}=a R_{0} \omega \Re\left\{\hat{v}_{r}(\hat{r}, \hat{z}) e^{i \omega t}\right\}, \\
v_{z}=a H_{0} \omega \Re\left\{\hat{v}_{z}(\hat{z}) e^{i \omega t}\right\},
\end{gathered}
$$

\footnotetext{
${ }^{1}$ Commercial version of MFR available from GBC Scientific Equipment, Ltd., Dandenong, Victoria, Australia, www.gbcsci.com
}

$$
p=p_{\mathrm{atm}}+\frac{a \omega R_{0}^{2}}{H_{0}^{2}} \Re\left\{\eta^{*} \hat{p}(\hat{r}, \hat{z}) e^{i \omega t}\right\} .
$$

Here, $p_{\text {atm }}$ is the atmospheric pressure. Using Eqs. (11)-(15), the following dimensionless complex equations of continuity and motion are obtained:

$$
\begin{gathered}
\frac{1}{\hat{r}} \frac{\partial}{\partial \hat{r}}\left(\hat{r} \hat{v}_{r}\right)+\frac{\partial \hat{v}_{z}}{\partial \hat{z}}=0, \\
\operatorname{Re} \hat{v}_{r}=-\frac{\partial \hat{p}}{\partial \hat{r}}+\left[\Lambda^{2} \frac{\partial}{\partial \hat{r}}\left(\frac{1}{\hat{r}} \frac{\partial}{\partial \hat{r}}\left(\hat{r} \hat{v}_{r}\right)\right)+\frac{\partial^{2} \hat{v}_{r}}{\partial \hat{z}^{2}}\right], \\
\Lambda^{2} \operatorname{Re} \hat{v}_{z}=-\frac{\partial \hat{p}}{\partial \hat{z}}+\left[\Lambda^{4} \frac{1}{\hat{r}} \frac{\partial}{\partial \hat{r}}\left(\hat{r} \frac{\partial \hat{v}_{z}}{\partial \hat{r}}\right)+\Lambda^{2} \frac{\partial^{2} \hat{v}_{z}}{\partial \hat{z}^{2}}\right] .
\end{gathered}
$$

Here, $\rho$ is the density, and we define the Reynolds number as

$$
\operatorname{Re}=\frac{i \rho \omega H_{0}^{2}}{\eta^{*}}
$$

Note that various definitions of Re have been used for oscillatory squeeze flow (OSF). Field et al. [18] define it as a real number and use $a H_{0}$ as the characteristic length to obtain $\operatorname{Re}=\rho \omega a H_{0}^{2} / \eta^{*}$. Debbaut and Thomas [21] do not define a Reynolds number but introduce the number $\alpha^{2}=i \omega \rho / \eta^{*} . \alpha$ has dimensions of $m^{-1}$ but always appears in the group $\alpha^{2} H_{0}^{2}$, which is dimensionless and equal to the Re defined here [22].

Assuming that material planes remain parallel to the plates, we find that $\hat{v}_{z}$ is independent of $\hat{r}$ so that mass conservation Eq. (16) yields

$$
\begin{gathered}
\hat{v}_{r}=-\frac{1}{2} f^{\prime}(\hat{z}) \hat{r}, \\
\hat{v}_{z}=f(\hat{z}) .
\end{gathered}
$$

Here, $f(\hat{z})$ is a function that varies only with $\hat{z}$, and $f^{\prime}(\hat{z})$ is the derivative. Inserting Eqs. (20) and (21) into Eqs. (18) and (19), we obtain

$$
\begin{aligned}
& \frac{1}{2} \operatorname{Re} \hat{r} f^{\prime}=\frac{\partial \hat{p}}{\partial \hat{r}}+\frac{1}{2} \hat{r} f^{\prime \prime \prime}, \\
& \Lambda^{2} \operatorname{Re} f=-\frac{\partial \hat{p}}{\partial \hat{z}}+\Lambda^{2} f^{\prime \prime} .
\end{aligned}
$$

Performing cross differentiation, the pressure can be eliminated, and the following expression is obtained:

$$
\operatorname{Re} f^{\prime \prime}=f^{\prime \prime \prime \prime}
$$

With boundary conditions

$$
\begin{gathered}
f(1)=1, \quad f(0)=0, \\
f^{\prime}(1)=0, f^{\prime}(0)=0 .
\end{gathered}
$$


Proceeding from here, it is beneficial to identify other simplifying assumptions that might apply. In particular, postulate

(1) If inertia can be neglected, i.e., if $\mathrm{Re} \ll 1$. In this case, the flow can be regarded as a creep flow, and the left hand side of Eqs. (22)-(24) can be set equal to 0 .

(2) If $\Lambda \ll 1$, the lubrication approximation can be used so that all terms containing any order of $\Lambda$ can be neglected in Eq. (23).

We now examine the effect of making either of these assumptions. In all cases, we assume a no-slip boundary condition between the fluid and the plates, although we discuss briefly the effect of a slip or partial-slip boundary condition below.

\section{A. Negligible inertia}

We first assume small Re so that inertia is negligible compared to viscous effects, and Eq. (24) reduces to

$$
f^{\prime \prime \prime \prime}=0 \text {. }
$$

Using boundary conditions (25) and (26), one obtains

$$
f=-2 \hat{z}^{3}+3 \hat{z}^{2}
$$

The radial and axial stresses (physical components) are then found to be

$$
\begin{aligned}
\sigma_{r r} & =\frac{a \omega R_{0}^{2}}{H_{0}^{2}} \Re\left\{\eta^{*} \hat{\sigma}_{r r} e^{i \omega t}\right\}, \text { where } \hat{\sigma}_{r r}=6 \Lambda^{2}\left(\hat{z}^{2}-\hat{z}\right), \\
\sigma_{z z} & =\frac{a \omega R_{0}^{2}}{H_{0}^{2}} \Re\left\{\eta^{*} \hat{\sigma}_{z z} e^{i \omega t}\right\}, \text { where } \hat{\sigma}_{z z}=-12 \Lambda^{2}\left(\hat{z}^{2}-\hat{z}\right) .
\end{aligned}
$$

Returning to the complex, dimensionless equations of motion [Eqs. (22) and (23)], we can neglect terms containing $\mathrm{Re}$ and set the right hand sides to 0 . Inserting the expression for $f(\hat{z})$, one obtains

$$
\begin{gathered}
0=\frac{\partial \hat{p}}{\partial \hat{r}}-6 \hat{r} \\
0=-\frac{\partial \hat{p}}{\partial \hat{z}}+\Lambda^{2}(-12 \hat{z}+6) .
\end{gathered}
$$

With the solution

$$
\hat{p}=3 \hat{r}^{2}+6 \Lambda^{2}\left(-\hat{z}^{2}+\hat{z}\right)+\hat{p}_{0}
$$

Here, $\hat{p}_{0}$ is an integration constant. The most intuitive boundary condition would be to set the pressure equal to the atmospheric pressure at the outer $\operatorname{rim}(\hat{r}=1)$. However, this procedure is problematic since $\hat{p}$ depends on $\hat{z}$. One way to deal with this issue is to set the $z$-averaged pressure $p=p_{\text {atm }}$ [1]. Another approach is to set up a force balance at $(\hat{r}, \hat{z})=(1,1 / 2)$. Here, only the stress in the radial direction needs to be considered, leading to the following expressions:

$$
\begin{gathered}
0=\hat{p}\left(1, \frac{1}{2}\right)-\hat{\sigma}_{r r}\left(1, \frac{1}{2}\right), \\
\Rightarrow \hat{p}_{0}=-3\left(1+\Lambda^{2}\right) .
\end{gathered}
$$

Hence, the complete expression for $\hat{p}$ is

$$
\hat{p}=-3\left(1-\hat{r}^{2}+\Lambda^{2}\right)+6 \Lambda^{2}\left(-\hat{z}^{2}+\hat{z}\right) .
$$

In terms of physical components, we have

$$
\begin{aligned}
p-p_{\text {atm }}= & \frac{a \omega}{H_{0}^{2}}\left[-3\left(R_{0}^{2}-r^{2}+H_{0}^{2}\right)+6\left(-z^{2}+H_{0} z\right)\right] \\
& \times \Re\left\{\eta^{*} e^{i \omega t}\right\} .
\end{aligned}
$$

The expression $p-p_{\text {atm }}-\sigma_{z z}$ can then be integrated over either the top or bottom plate, i.e., $z=H_{0}$ or $z=0$, to give the normal force $(F)$ on the top plate.

Another approach is to use another fact at our disposal to deal with this tricky boundary condition that will allow us to completely avoid it. This is to use the macroscopic mechanical energy balance adapted to linear viscoelastic liquids [23, Sec. 7.8].

$$
\boldsymbol{F} \dot{H}=\int_{v} \boldsymbol{\sigma}: \nabla \boldsymbol{v} d V
$$

Since $\dot{H}$ is completely in phase with $v$, it follows that $\boldsymbol{F}$ must be in phase with $\boldsymbol{\sigma}$ and is given by

$$
\boldsymbol{F}=\left(a H_{0} \omega\right)^{-1} \int_{v} \phi_{V} d V \Re\left\{\eta^{*} e^{i \omega t}\right\},
$$

where

$$
\phi_{V}=2\left[\left(\frac{d \bar{v}_{r}}{d r}\right)^{2}+\left(\frac{d \bar{v}_{z}}{d z}\right)^{2}\right]+\left[\frac{d \bar{v}_{r}}{d z}\right]^{2}
$$

Thus, the volume integral over $\phi_{V}$ gives

$$
\begin{aligned}
\int_{v} \phi_{V} d V= & 2 \pi\left(a \omega R_{0}\right)^{2} H_{0} \Re\left\{\int _ { \hat { z } = 0 } ^ { 1 } \int _ { \hat { r } = 0 } ^ { 1 } \left(2\left[\left(\frac{d \hat{v}_{r}}{d \hat{r}}\right)^{2}+\left(\frac{d \hat{v}_{z}}{d \hat{z}}\right)^{2}\right]\right.\right. \\
& \left.\left.+\left[\frac{R_{0}}{H_{0}} \frac{d \hat{v}_{r}}{d \hat{z}}\right]^{2}\right) \hat{r} d \hat{r} d \hat{z}\right\} \\
= & \frac{3 \pi(a \omega)^{2} R_{0}^{4}}{2 H_{0}}\left[1+2\left(\frac{H_{0}}{R_{0}}\right)^{2}\right]
\end{aligned}
$$

The final expression for $F$ is obtained by inserting this into Eq. (39) and we get

$$
\Rightarrow F=\frac{3 \pi a \omega R_{0}^{4}}{2 H_{0}^{2}} \Re\left\{\eta^{*} e^{i \omega t}\right\}\left[1+2\left(\frac{H_{0}}{R_{0}}\right)^{2}\right] .
$$

Inserting $i \omega \eta^{*}=\left(G^{\prime}+i G^{\prime \prime}\right)$ and taking the real part of all terms results in following: 


$$
F=\frac{3 \pi a R_{0}^{4}}{2 H_{0}^{2}}\left(G^{\prime} \sin \omega t+G^{\prime \prime} \cos \omega t\right)\left[1+2\left(\frac{H_{0}}{R_{0}}\right)^{2}\right] .
$$

The factor $\left[1+2\left(H_{0} / R_{0}\right)^{2}\right]$ weighs the shear contribution to the normal force compared to the extensional contribution. If $H_{0} / R_{0} \gg 1$, the second term in brackets dominates the first, and the flow can be considered uniaxial extension. Hence, the relation reduces to the well-established expression for the normal force on the top plate for uniaxial viscoelastic extensional flow [3]

$$
F=3 \pi a R_{0}^{2}\left(G^{\prime} \sin \omega t+G^{\prime \prime} \cos \omega t\right) .
$$

But if $H_{0} / R_{0} \ll 1$, the first term in Eq. (43) dominates the second so that the deformation is dominated by shear, and Eq. (43) reduces to

$$
F=\frac{3 \pi a R_{0}^{4}}{2 H_{0}^{2}}\left(G^{\prime} \sin \omega t+G^{\prime \prime} \cos \omega t\right) .
$$

This equation can be derived from the Stefan equation [Eq. (1)] using Pipkin's correspondence principle [16], as shown originally derived by Phan-Thien [17]. Phan-Thien's final expression contained an error later corrected by Field et al. [18] who give the correct form shown below:

$$
F^{*}=\frac{3 \pi R_{0}^{4} \eta^{*}}{2 H_{0}^{3}} \frac{d H^{*}}{d t}
$$

The price paid for this apparent simplicity is that $F^{*}$ and $H^{*}$ are now complex functions of time related to the force and the plate separation by $F=\Re\left\{F^{*}\right\}$ and $H=\Re\left\{H^{*}\right\}$, respectively.

The very simple relations, Eqs. (45) and (46), are often applied to OSF as the aspect ratio in most cases is very small, and hence, the deformation can be safely assumed to be dominated by shear. However, if inertia is non-negligible, a correction is needed as is shown below.

\section{B. Lubrication approximation}

Now we assume a small aspect ratio and apply the lubrication approximation (assumption 2 above). However, this does not lead to any simplification of Eq. (24) which we focus on initially in the following. The full solution of Eq. (24), which was derived by Bell et al. [22], is rather complicated and thus has limited practical utility. Instead it is beneficial to consider the perturbation solution in Re. We represent the full solution $f$ as a power series in $\operatorname{Re}$

$$
f(\operatorname{Re}, \hat{z})=f_{0}+\operatorname{Re} f_{1}+O\left(\operatorname{Re}^{2}\right) .
$$

So far, we have limited our analysis to a first order approximation in Re, and the approach is the same, but it becomes increasingly messy at higher orders. Inserting Eq. (47) into Eq. (24) gives

$$
\operatorname{Re} f_{0}^{\prime \prime}=f_{0}^{\prime \prime \prime \prime}+\operatorname{Re} f_{1}^{\prime \prime \prime \prime}+O\left(\operatorname{Re}^{2}\right) \text {. }
$$

Collecting terms of equal order in Re yields following system of equations:

$$
\begin{gathered}
f_{0}^{\prime \prime \prime \prime}=0, \\
f_{0}^{\prime \prime}-f_{1}^{\prime \prime \prime \prime}=0 .
\end{gathered}
$$

With the following boundary conditions:

$$
\begin{aligned}
& f_{0}(1)=1, \quad f_{0}(0)=0, \\
& f_{0}^{\prime}(1)=0, \quad f_{0}^{\prime}(0)=0, \\
& f_{1}(1)=0, \quad f_{1}(0)=0, \\
& f_{1}^{\prime}(1)=0, \quad f_{1}^{\prime}(0)=0 .
\end{aligned}
$$

Terms of $O\left(\mathrm{Re}^{2}\right)$ are disregarded, as they are assumed to be negligibly small. We see that Eq. (49) is identical to Eq. (27). This is expected, since both are of order $O\left(\operatorname{Re}^{0}\right)$, and it obviously follows that both have the same solution. This solution is subsequently applied when solving for $f_{1}$ and we obtain

$$
\begin{gathered}
f_{0}=-2 \hat{z}^{3}+3 \hat{z}^{2}, \\
f_{1}=-\frac{1}{10} \hat{z}^{5}+\frac{1}{4} \hat{z}^{4}-\frac{1}{5} \hat{z}^{3}+\frac{1}{20} \hat{z}^{2} .
\end{gathered}
$$

Until now, no simplifications based on lubrication have been made. However, returning to the equations of motion [Eqs. (22) and (23)], the lubrication approximation can be applied by eliminating all terms containing any order of $\Lambda$. If we also insert the 1 st order perturbation solution $f=f_{0}+\operatorname{Re} f_{1}+O\left(\operatorname{Re}^{2}\right)$, we obtain

$$
\begin{gathered}
\frac{d \hat{p}}{d \hat{r}}=-\frac{1}{2} \hat{r}\left[f_{0}^{\prime \prime \prime}+\operatorname{Re}\left(f_{1}^{\prime \prime \prime}-f_{0}^{\prime}\right)\right] \\
\frac{d \hat{p}}{d \hat{z}}=0 .
\end{gathered}
$$

We see that in this case $\hat{p}$ is independent of $\hat{z}$. Inserting the expressions for $f_{0}$ and $f_{1}$ in Eq. (57) yields the following differential equation:

$$
\frac{d \hat{p}}{d \hat{r}}=6 \hat{r}\left(1+\frac{1}{10} \operatorname{Re}\right)
$$

whose solution is

$$
\hat{p}=3 \hat{r}^{2}\left(1+\frac{1}{10} R e\right)+\hat{p}_{1} .
$$

Here, $\hat{p}_{1}$ is a dimensionless integration constant. At the rim, $\hat{r}=1$ and we apply the boundary condition that $p=p_{\text {atm }}$ leading to $\hat{p}=0$, and we obtain the following expression for $\hat{p}$ :

$$
\hat{p}=-3\left(1-\hat{r}^{2}\right)\left(1+\frac{1}{10} \operatorname{Re}\right) .
$$


In terms of physical components, the pressure is

$$
\begin{aligned}
p-p_{\mathrm{atm}}= & -\frac{3 a \omega R_{0}^{2}}{H_{0}^{2}}\left[1-\left(\frac{r}{R_{0}}\right)^{2}\right] \\
& \times \Re\left\{\eta^{*}\left(1+\frac{1}{10} \mathrm{Re}\right) e^{i \omega t}\right\} .
\end{aligned}
$$

The normal force $F$ is obtained by integration of $p-p_{\mathrm{atm}}$, and the following expression is obtained:

$$
\begin{gathered}
F=-2 \pi \int_{0}^{R_{0}}\left(p-p_{\text {atm }}\right) r d r \\
\Rightarrow F=\frac{3 \pi a \omega R_{0}^{4}}{2 H_{0}^{2}} \Re\left\{\eta^{*}\left(1+\frac{1}{10} \operatorname{Re}\right) e^{i \omega t}\right\}, \\
\Rightarrow F=\frac{3 \pi a R_{0}^{4}}{2 H_{0}^{2}}\left[\left(G^{\prime}-\frac{\rho \omega^{2} H_{0}^{2}}{10}\right) \sin \omega t+G^{\prime \prime} \cos \omega t\right] .
\end{gathered}
$$

Thus, the first order inertial correction only influences the storage modulus $G^{\prime}$. In situations where $\operatorname{Re} \approx 1$, an error of about $10 \%$ in $G^{\prime}$ is expected if the first order correction term is not included. This is in good agreement with results obtained by Debbaut and Thomas [21], who compared computational fluid dynamics (CFD) calculations to analytic expressions for systems where inertia plays a non-negligible role. Additionally, it should be noted that when $\operatorname{Re} \rightarrow 0$, Eq. (64) reduces to the creep solution for small aspect ratios [Eq. (45)] as it should. Debbaut and Thomas [21] also simulated nonlinear behavior using a Giesikus viscoelastic model to determine the effects of normal stress differences on the results. Their results revealed that normal stress differences affect data at high strains in a different way than nonlinearity affects data from rotational measurements.

The solution can also be derived for higher orders of $\mathrm{Re}$ as was done by Bell et al. [22], who extended earlier work of Phan-Thien [17] and Field et al. [18] to obtain the perturbation solution approximated to order $O\left(\mathrm{Re}^{2}\right)$ as follows:

$$
F=\frac{3 \pi a \omega R_{0}^{4}}{2 H_{0}^{2}} \Re\left\{\eta^{*}\left(1+\frac{1}{10} \operatorname{Re}+\frac{1}{8400} \operatorname{Re}^{2}\right) e^{i \omega t}\right\}
$$

From the prefactor of $\mathrm{Re}^{2}$, we see that the second order correction is needed only at very high Re. More specifically, an error of about $10 \%$ is expected when $\mathrm{Re} \approx 100$. In this high Reynolds number, the flow is dominated by inertia. This leaves one wondering whether the OSF-technique is of any use for these types of fluids as one has to extract a small fraction of the total normal force to obtain the viscous contribution.

With regard to the 1 st and 2nd-order correction terms, however, it is important to note that compared to a torsional rheometer, inertia for OSF is much less significant [22]. In a torsional rheometer, corrections for inertia are required at a much lower Re compared to the OSF-rheometer.

In closing, we note that the fundamental solution represented by the function $f_{0}$ allows us to get a more accurate estimate of the maximum shear strain at the outer rim. We find that the shear rate at the bottom plate is given by

$$
\dot{\gamma}_{r z}=-3 a \omega \frac{r}{H_{0}} \cos \omega t .
$$

The shear strain from some given initial time 0 to time $t$ is then

$$
\gamma(t, 0)=\int_{t^{\prime}=0}^{t} \dot{\gamma}_{r z} d t^{\prime}=-3 a \frac{r}{H_{0}} \sin \omega t .
$$

The maximum value is therefore $3 a / \Lambda$.

\section{Slip/partial slip boundary}

In this review, only a no-slip boundary condition for the top and bottom plates is considered. However, if the fluid does not fully stick to the wall, partial slip or complete slip will occur. This has been modeled for a nonoscillatory squeeze flow [24], where the degree of slip is accounted for from the slip velocity $\left(v_{s}\right)$ at the solid-liquid interphase

$$
v_{s}(r, H)=-\beta \sigma_{r z}(r, H) .
$$

Here, the slip coefficient $\beta$ is introduced as a lubrication measure, i.e., if $\beta=0$, a no-slip boundary condition applies, whereas complete slip is obtained in the limit of $\beta \rightarrow \infty$. If a full slip boundary condition is applied in the OSF model, one generates biaxial extension, as was mentioned in Sec. I. If fluid sticks only partially to the wall, the situation will be between the no-slip and full slip cases. The less slip, the greater will be the contribution of shearing deformation to the stress.

\section{INSTRUMENTS}

\section{A. Narrow gap squeeze flow devices}

Early work on the behavior of elastic fluids in squeeze flow was motivated by the question of whether elasticity enhanced the lubricating ability of a fluid squeezed between flat plates, and this was reviewed by Brindley et al. [25]. When the aspect ratio is sufficiently small, the lubrication approximation can be used, or if the viscosity is large, inertia can be ignored, and Eq. (45) can be used to interpret the data. A constant sample area is generally the preferred configuration, because if a smaller sample is used, its initial radius must be precisely known. Also, in the case of a constant volume sample, it is important that it be centered in the gap if symmetric loading is to be achieved. The principal advantage of the squeeze flow rheometer over rotational instruments is the simplicity of the apparatus. It has no air bearing and is much less expensive and easier to use. It might also find application as the central component of a high-throughput rheometer for evaluating experimental materials.

It was some years before an instrument to carry out such a test was described in a presentation by Whittingstall and van Arsdale [26] and a patent by Van Arsdale and Motivala [27]. Figure 2 shows the principle of operation.

The sample fills the gap, so this is a constant area device.

The first commercial instrument based on oscillatory squeeze flow was the CP20 Compressional Rheometer made by TA Instruments, a sketch of which is shown in Fig. 3. As in the Van Arsdale device, it is the sample area that is constant rather than its volume. The manufacture and sale of the 


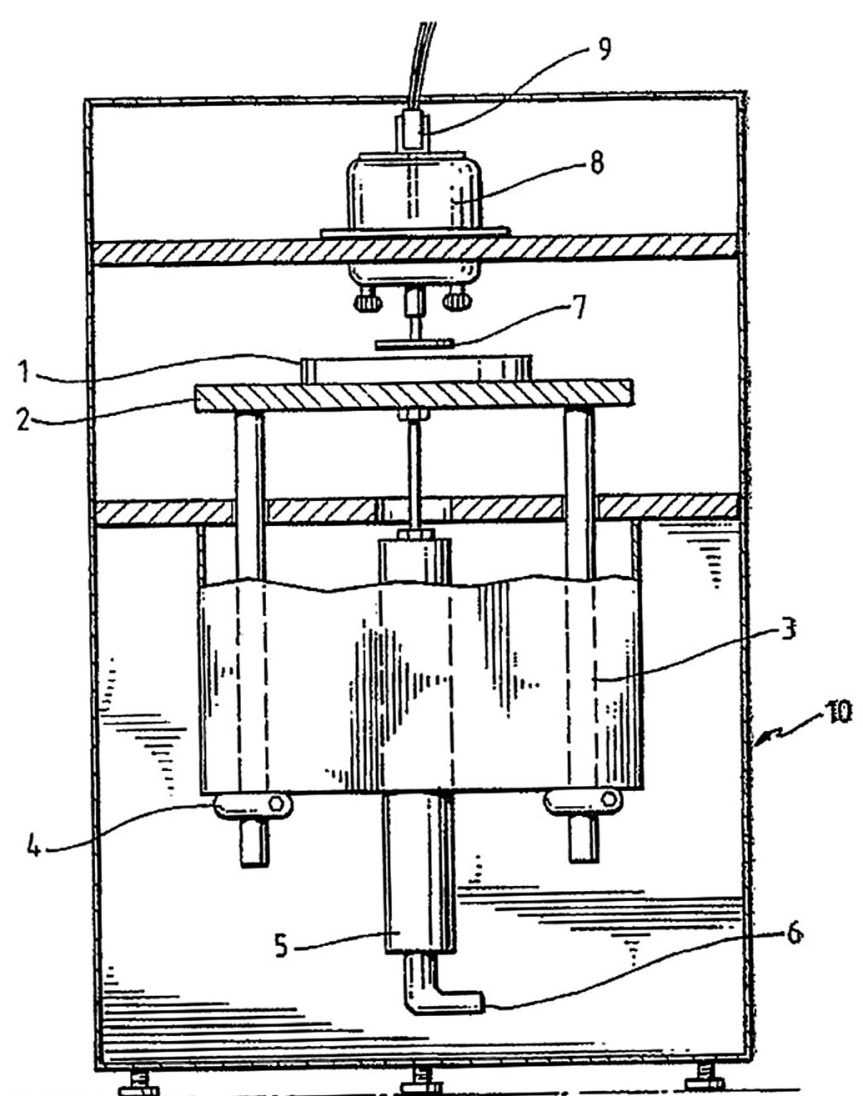

FIG. 2. Sketch showing essential elements of the device patented by van Arsdale and Motivala [27]. (1) Temperature-controlled stationary plate; (2) moveable base plate; (3) pneumatic linear actuator; (4) rings to limit vertical movement; (5) air cylinder; (6) air line; (7) oscillating plate; (8) shaker motor; (9) noncontacting displacement sensor; and (10) frame.

CP20 were later taken over by DB Scientific Instruments, which no longer exists, who called it the CP50 Compressional Rheometer. Figure 4 is a photo of this device. Note that the sample fills the gap under the upper plate.

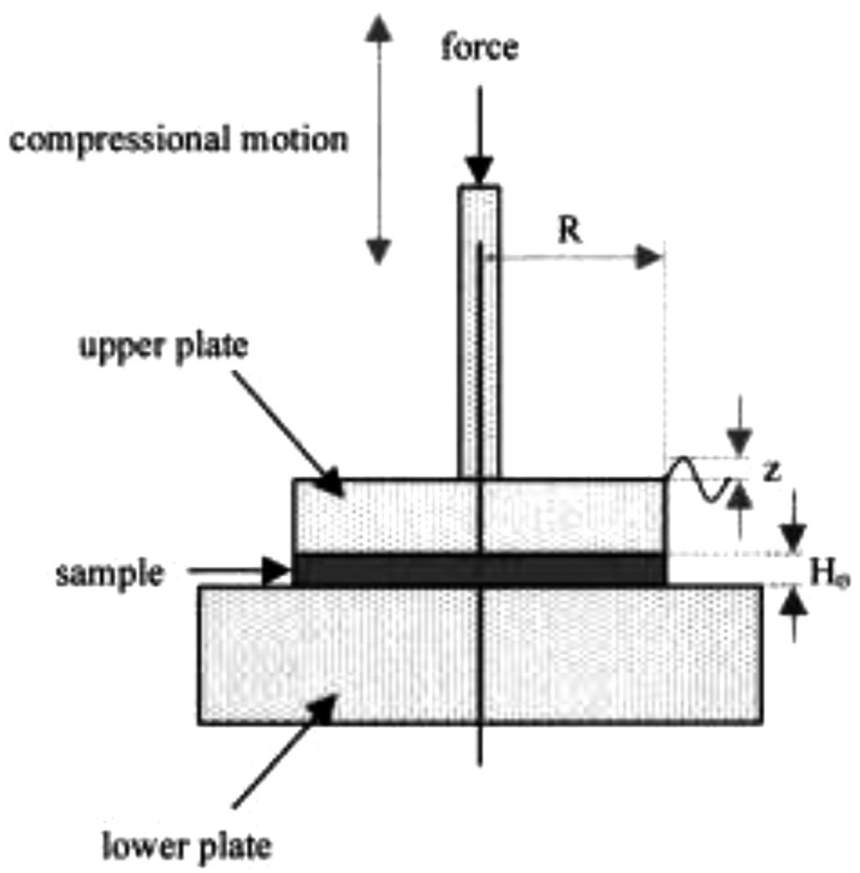

FIG. 3. The basic elements of the CP50 compressional rheometer.
Walberer and McHugh [28] used this device to study highly filled particle suspensions in polydimethylsiloxane.

Crassous et al. [29] described a device for high-frequency measurements driven by a piezoelectric axial vibrator. They were able to obtain storage and loss moduli for polymer solutions over a range of frequencies from 10 to $300 \mathrm{~Hz}$. In combination with a rotational rheometer and a set of torsional resonators, they were able to characterize a sample at frequencies from $10^{-3}$ to $77 \mathrm{kHz}$ without the use of timetemperature superposition. They used this set of devices to characterize an aqueous suspension of thermosensitive latex particles over the same range of frequencies.

\section{B. Nonsinusoidal deformation}

Nelson and Dealy [30] describe a number of nonsinusoidal waveforms that have been used for linear viscoelastic characterization, including multiple, superposed sine waves, an equistrain waveform designed to generate a uniform strain amplitude across a given band of frequencies, and a pseudorandom binary sequence. The advantage of this approach is that by use of a fast Fourier transform (FFT) program one can obtain results over a range of several decades of frequency in a single test. Nelson and Dealy [30] discussed several problems that arise in the use of the discrete Fourier transform to analyze the results.

Field et al. [18] employed pseudorandom squeezing in the device sketched in Fig. 5. A FFT is used to extract the frequency-dependent moduli. A characterization over two decades of frequency is obtained in a very short time.

A commercial version of this instrument is called the micro-Fourier rheometer (MFR), because it can deal with samples as small as $100 \mu \mathrm{l}$. Figure 6 is a photo of this device.

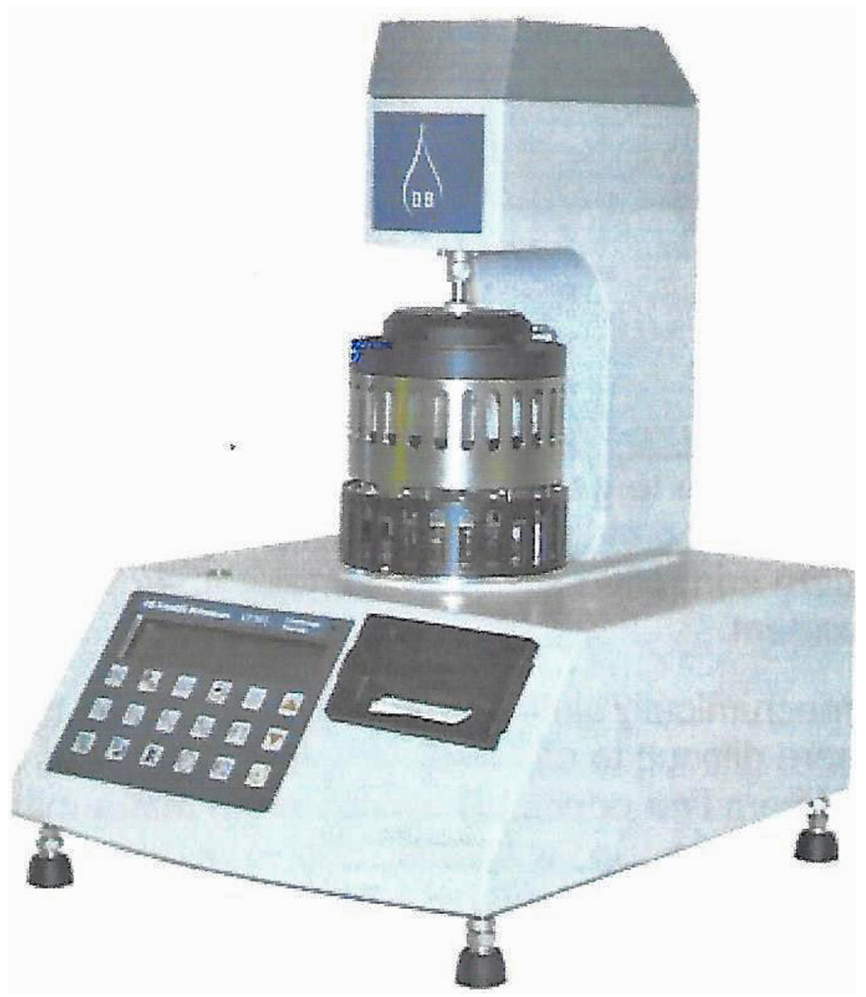

FIG. 4. Photo of the CP20 compressional rheometer. 


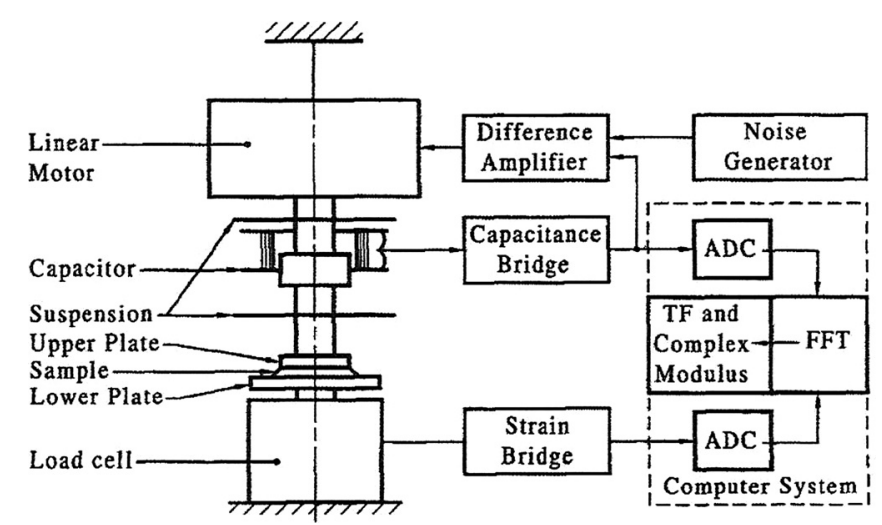

FIG. 5. Essential elements of the MFR of Field et al. [18].

The input is random noise of amplitude $n$ as shown below, where $\varepsilon \ll 1$

$$
h(t)=H_{0}[1+\varepsilon n(t)]=H_{0}+h^{\prime}(t) .
$$

The noise is described by its spectral representation [31]

$$
n(t)=\int_{-\infty}^{\infty} e^{i \omega t} d Z(\omega)
$$

where $d Z(\omega)$ is a random function with zero means and a known correlation.

Phan-Thien et al. [20] examined the effects of inertia on the operation of this instrument. Because a complete characterization can be carried out quickly, the MFR can be used to track rapid changes in properties. This device has been used, for example, to study particle suspensions [32] and dental composite resins [33].

Sakai [34] modified a MFR for use with fluids employed in the manufacture of micromechanical electric systems. Of special interest here are interactions between dispersed particles and dissolved polymers. The fluid response is primarily

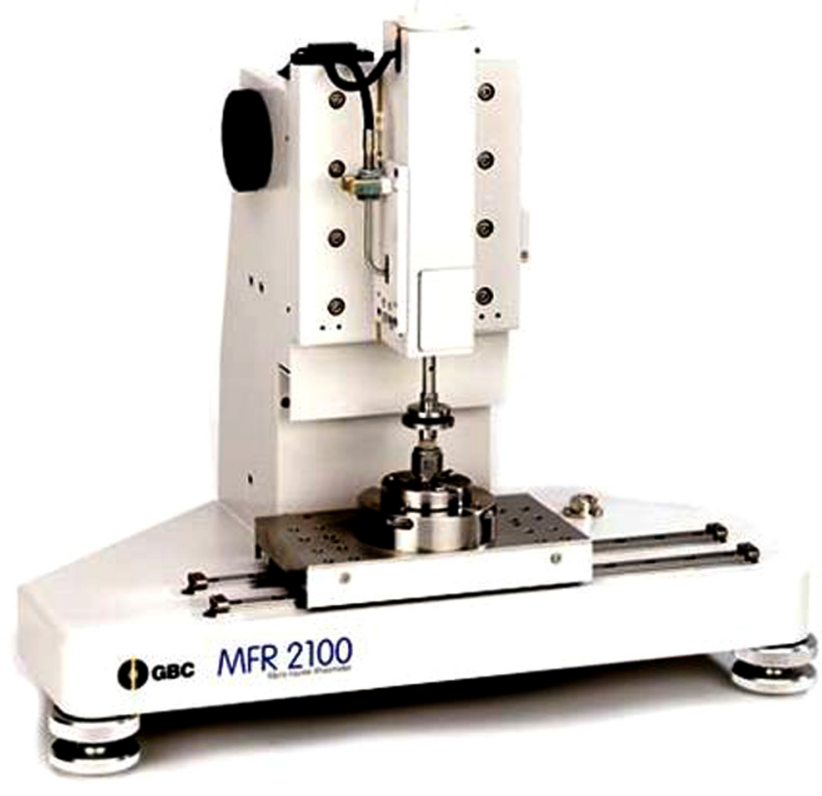

FIG. 6. Photo of the MFR2100 MFR of GBC scientific equipment.

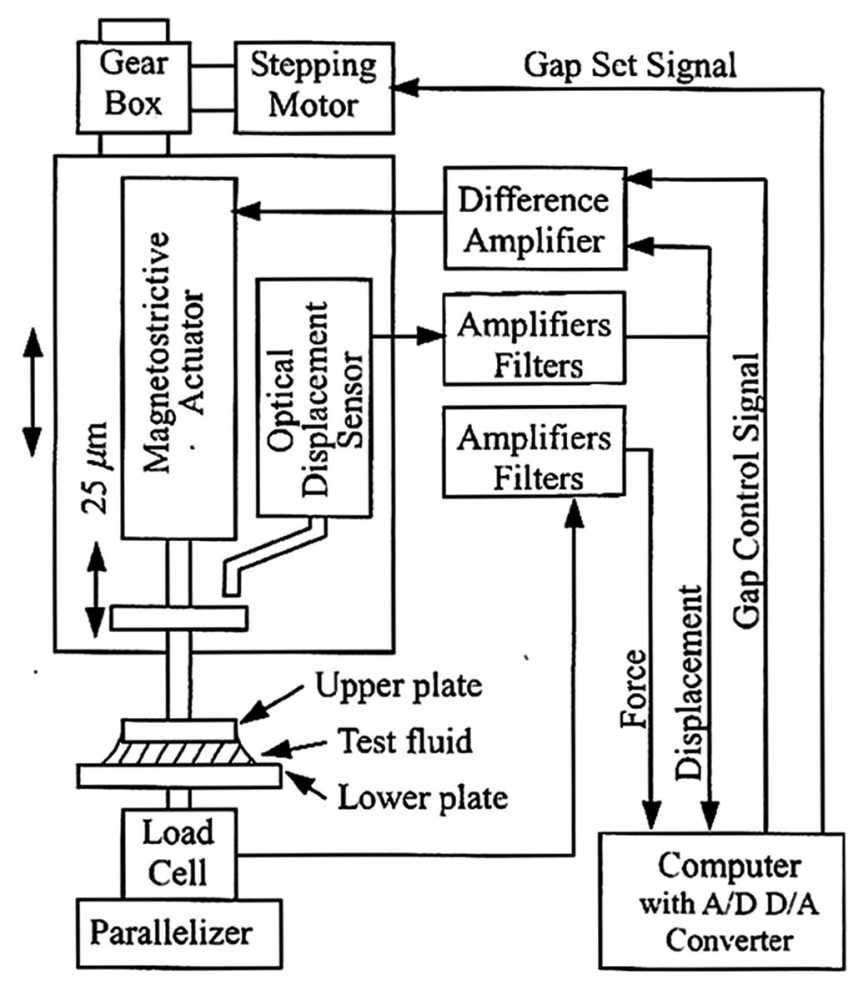

FIG. 7. The MFR of Sakai [34].

viscous, but elasticity is still important, and a major challenge is detecting the very small phase angle of the response to oscillatory squeezing. Figure 7 shows this apparatus, in which the amplitude of the deformation is only $25 \mu \mathrm{m}$.

Another device based on the same basic principle was designed for quality assurance in manufacturing processes [35-37]. ${ }^{2}$ It measures the state of a fluid while it is in process, either in an in-line or side-stream installation.

\section{Filament stretching rheometer}

The filament stretching rheometer (FSR) was originally developed for the characterization of polymeric liquids in a nonlinear uniaxial extension [38,39]. It is similar to OSF instruments in the sense that it consists of a top plate and a bottom plate between which the sample is placed. As shown in Fig. 8, the top plate moves in an axial direction while the bottom plate remains fixed and is mounted on a strain-gauge load cell.

The major difference between OSF instruments described above and the FSR is that in the latter the deformation is not measured from the position of the top plate but across the sample midplane using a laser-sheet. Whereas the aspect ratio is about unity, it is very small in the OSF devices described in Secs. III A and III B. For use in OSF, the topplate undergoes oscillatory displacement to impose a sinusoidal compression on the sample, but in the FSR, a laser moves in-phase at half the speed of the top plate, in order to

${ }^{2}$ Commercial version available from Rheology Solutions, Bacchus Marsh, VIC, Australia, www.rheologysolutions.com 


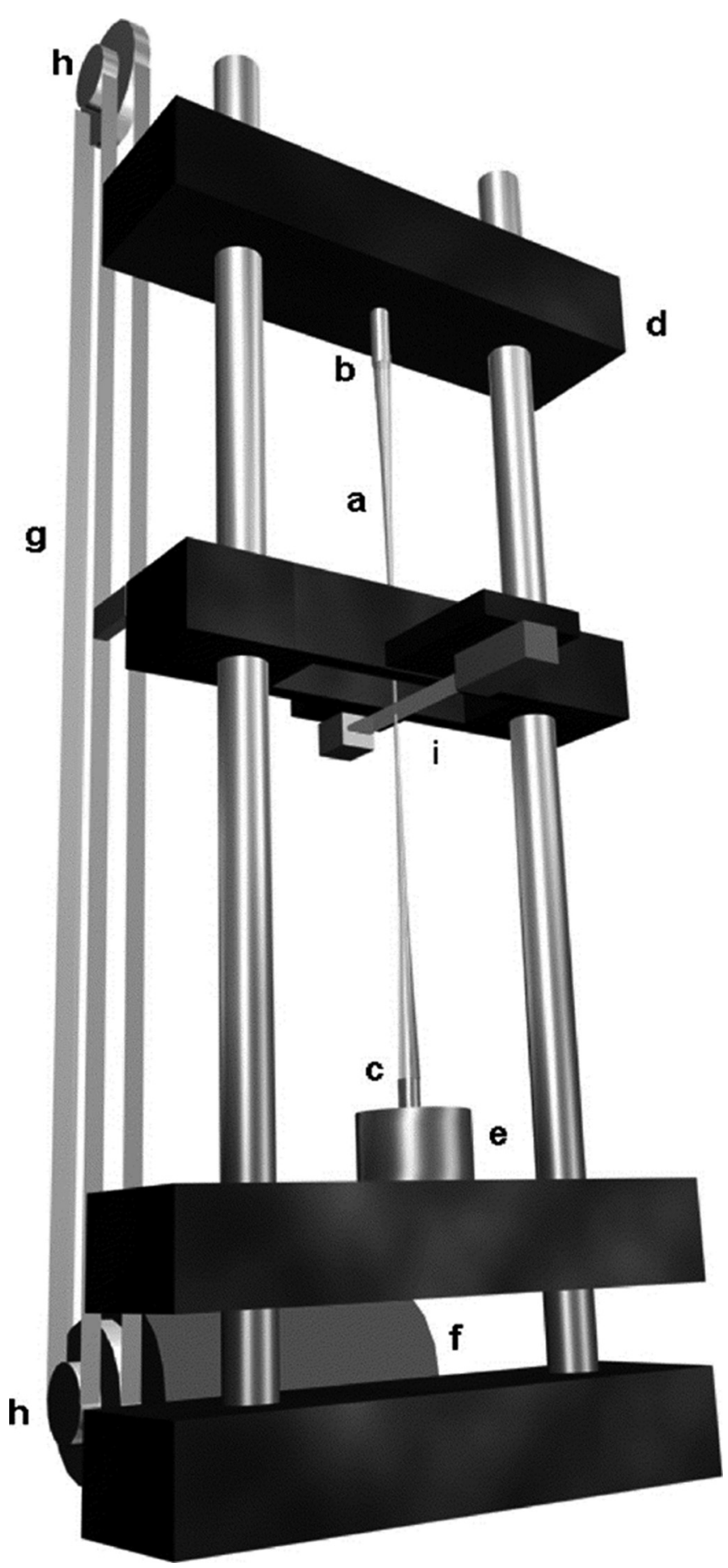

FIG. 8. Sketch of the FSR configures for uniaxial extension. (a) The filament, (b) top plate, (c) bottom plate, (d) top plate support, (e) force transducer, (f) motor, (g) belts, (h) gearing, and (i) laser micrometer [39].

remain at the level of the sample midplane. The relation between the top plate position, $H(t)$, and the sample diameter at the midplane, which is $D=D_{0}(1+b \sin \omega t)$ with $b D_{0}$ being the amplitude [3], is given by

$$
\begin{gathered}
\frac{\dot{H}}{H_{0}}=-\frac{4}{3} \frac{1}{D_{0}} \frac{d D}{d t}, \\
\Rightarrow b=-\frac{3}{4} a .
\end{gathered}
$$

This relation can simply be substituted into, e.g., Eq. (43) to give $F$ as a function of deformation across the midplane rather than the top plate

$$
F=-\frac{2 \pi b R_{0}^{4}}{H_{0}^{2}}\left(G^{\prime} \sin \omega t+G^{\prime \prime} \cos \omega t\right)\left[1+2\left(\frac{H_{0}}{R_{0}}\right)^{2}\right]
$$

This way of measuring deformation overcomes the problem of load cell compliance.

Using the FSR for linear characterization enables linear oscillatory and nonlinear uniaxial characterization using a single instrument with a single sample. More specifically, uniaxial extension can be performed directly after linear characterization. As the aspect ratio $(\Lambda)$ of the samples that are used for uniaxial extension in the FSR are much higher $(\Lambda \approx 1)$ compared to other OSF techniques, a significant extensional contribution to the stress will be present. Consequently, the lubrication approximation cannot be used. In many cases, Eq. (43) will be sufficient as inertia is rarely a factor with the very viscous fluids characterized in uniaxial extension using the FSR.

At the present time, the FSR in the OSF mode has been used to study only one set of polystyrene melts [40]. Figure 9 shows dynamic moduli data obtained using both the FSR and an Ares G2 for a commercial polystyrene with $M_{w}=$ $230 \mathrm{~kg} / \mathrm{mol}$ (PS-230k). Data from the two instruments agree very well, although there is a small vertical shift between the two sets of data.

\section{APPLICATIONS}

\section{A. Polymer melts and solutions}

Polymer melts and solutions have been used in several studies to test OSF technique. One advantage of using polymeric liquids is that inertial effects can be brought out or suppressed as desired due to the high degree of rheological tunability that these materials offer. This approach was employed by [22] to investigate fluids in which inertia was

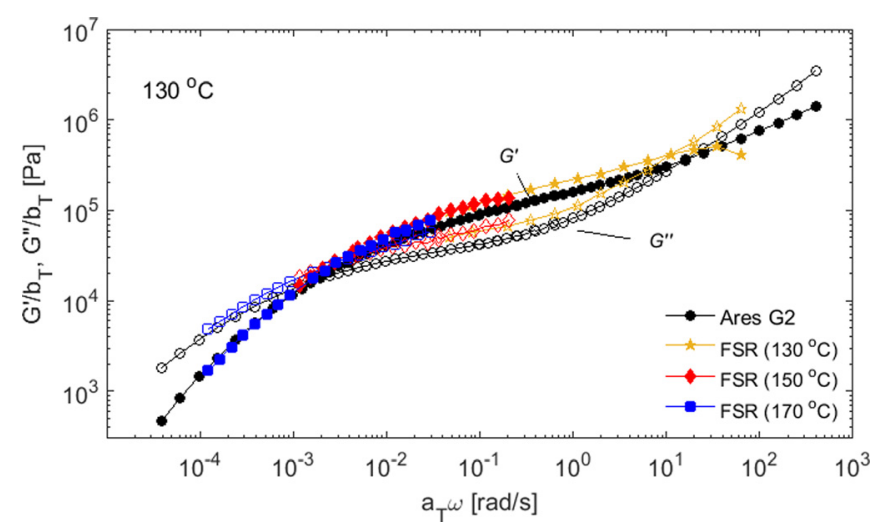

FIG. 9. $G^{\prime}$ (closed symbols) and $G^{\prime \prime}$ (open symbols) of a commercial PS$230 \mathrm{~K}$ at $130^{\circ} \mathrm{C}$. Data points obtained using the FSR [Eq. (74)] at various temperatures that has been shifted to $130^{\circ} \mathrm{C}$ using shift factors $a_{T}$ and $b_{T}$, respectively. Diamonds: FSR $\left(150^{\circ} \mathrm{C}\right)$; Stars: FSR $\left(150^{\circ} \mathrm{C}\right)$; Squares: FSR $\left(170^{\circ} \mathrm{C}\right)$. Circles show data obtained using a commercial torsional Ares G2 rheometer [40]. 
negligible and fluids for which inertial corrections to the 1 st or 2 nd order were needed.

Bulk polymers usually have very high viscosities and are thus useful to verify Eqs. (43) and (45), in which inertial effects are neglected. Figure 10 shows storage and loss moduli of a highly viscous polydimethyl sulfate measured using a conventional torsional rheometer (AR 2000) and an OSF rheometer with several gap heights. The OSF results were calculated without correcting for inertia. As seen, the results between the two techniques are in very good agreement, which confirms that a correction for inertia is not needed for highly viscous fluids. Some concentrated polymer solutions, for example, the $30 \mathrm{wt}$. \% polystyrene solution studied by Crassous et al. [29], were sufficiently viscous that an inertial correction was not required. In general, this makes characterization of thick fluids very simple and convenient, and OSF is an excellent means for characterization for such fluids. Bell et al. [22] showed that compared to a conventional torsional rheometer, one can operate at much higher Re in the OSF rheometer before a correction for inertia is required.

Several OSF-studies used a polymer solution called the "A1 fluid." It is a thoroughly tested standard fluid [41] and was used both to validate the accuracy of the OSF rheometer and as a model material in numerical simulations to evaluate the influence of inertia and various boundary conditions $[21,22]$. The A1 fluid is a $2 \%(\mathrm{w} / \mathrm{v})$ polyisobutylene solution in a mixture of cis and trans decalin. Dilution of polymers will normally lower the viscosity, and the inertial effects will become more significant but far from dominant. Results performed both on the A1 fluid and other polymer solutions
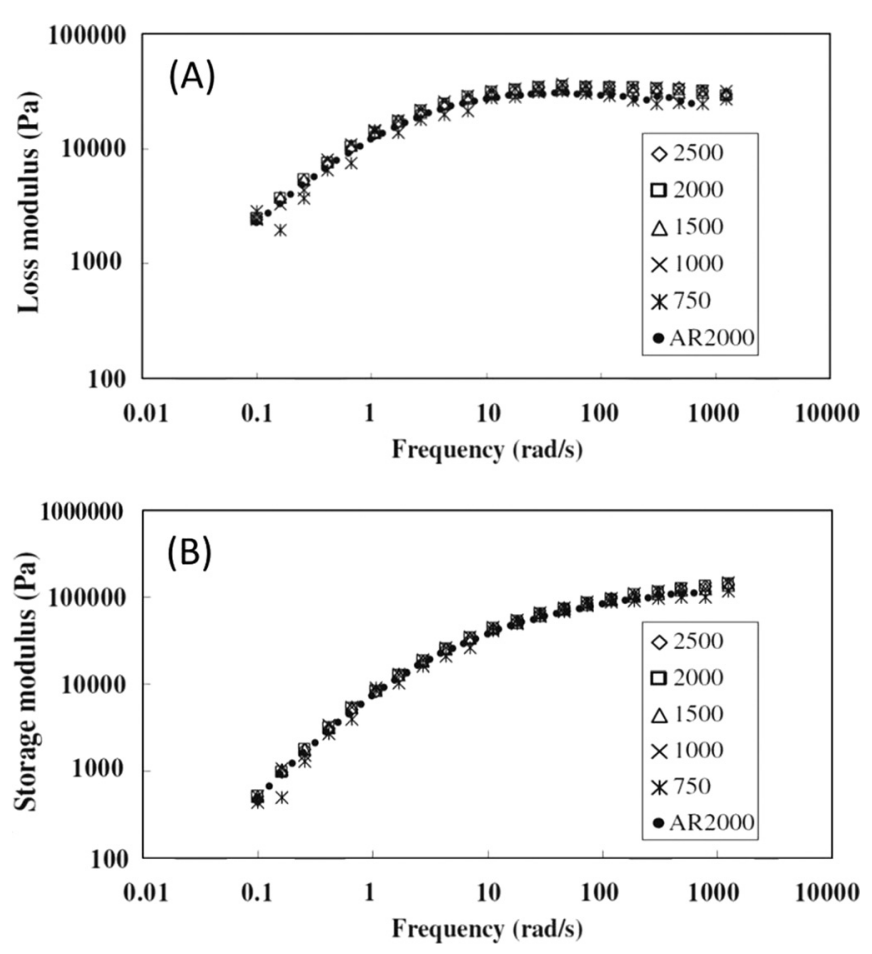

FIG. 10. Rheological characterization of a polydimethyl sulfate solution using the CP50 compressional rheometer; no inertial correction was applied [22]. Symbols indicate various gap heights in microns. (O) shows results obtained using a commercial torsional AR2000 rheometer. (A) The loss modulus and (B) the storage modulus. such as hyaluronic acid in methylcellulose proved to be in very good agreement with data from commercial rheometers, and in all cases, only an inertial approximation to the $1 \mathrm{st}$ order was needed $[20,28]$. The only material for which a 2 nd order inertial correction was needed was a $1 \%$ polyacrylamide solution in water [22]. This system was specifically designed to have a low viscosity in order to investigate the 2nd order inertia correction.

\section{B. Other materials}

See et al. [32] used a MFR shown in Fig. 5 to study the behavior of a suspension of spheres in a polymeric liquid. Walberer and McHugh [28] used the CP-20 rheometer to study the effects of polymer molecular weight and filler content in a glass-bead-filled polydimethyl siloxane. See and Nguyen [42] used a prototype MFR to monitor the curing of an epoxy resin and a plastic putty. Peng and Zhu [43], See et al. [44], and Lee and Wen [45] used OSF to study the behavior of an electrorheological fluid, which they modeled as a Bingham plastic with continuous modification. Kuzhir et al. [46] studied a suspension of magnetic particles, and Jiang et al. [33] used a MFR to track the curing of dental composite resin cements. They found that the response was linear during the earlier stages of cure but became nonlinear at very small strain amplitude.

\section{Large-amplitude squeeze flow}

Kim and Ahn $[47,48]$ studied combined oscillatory rotation and squeezing flow, which they called "dynamic helical squeeze flow" (DHSQ). They modified a Rheometric RMS800 rheometer to make it possible to do normal torsional flow, OSF, and combined DHSQ flow. In their 2012 paper, they compared the strain for the onset of nonlinear viscoelasticity for DHSQ and OSF for a polybutene with $M_{\mathrm{w}}=920 \mathrm{~g} / \mathrm{mol}$ and found that the values were 0.02 and 1.0 , respectively. The nonlinearities that arise at higher strains for both flows are not related to those observed in largeamplitude oscillatory shear in rotational instruments, as the deformations are quite different in the two flows.

\section{NOMENCLATURE}

\section{Symbol}

a Oscillation amplitude relative to the sample height, Eq. (6) (-)

$b$ Oscillation amplitude relative to the sample diameter, Eq. (73) (-)

$D$ Sample diameter $2 R(\mathrm{~m})$

$D_{0} \quad$ Mean sample diameter $2 R_{0}(\mathrm{~m})$

$f$ Dimensionless complex function of $\hat{z}$, Eqs. (21) and (22) (-)

$F \quad$ Normal force exerted on a plane by a fluid, Fig. 1 $\left(\mathrm{kg} \mathrm{m} \mathrm{s}^{-2}\right)$

$f_{0}$ Dimensionless complex function of $\hat{z}$ in the perturbation solution $(f)$, contributing an order $O\left(\operatorname{Re}^{0}\right)$ to the full solution of $f$, Eq. (47) (-) 
$F_{0} \quad$ Constant compressive force exerted on a plane by a fluid $\left(\mathrm{kg} \mathrm{m} \mathrm{s}^{-2}\right)$

$f_{1}$ Dimensionless complex function of $\hat{z}$ in the perturbation solution $(f)$, contributing an order $O\left(\operatorname{Re}^{1}\right)$ to the full solution of $f$, Eq. (47) (-)

$F^{*} \quad$ Complex function related to normal force $F=$ $\Re\left\{F^{*}\right\}\left(\mathrm{kg} \mathrm{m} \mathrm{s}^{-2}\right)$

$G^{*} \quad$ Complex modulus $G^{\prime}+i G^{\prime \prime}(\mathrm{Pa})$

$G^{\prime} \quad$ Storage modulus $(\mathrm{Pa})$

$G^{\prime \prime} \quad$ Loss modulus (Pa)

$H \quad$ Sample height Fig. 1 (m)

$\dot{H} \quad$ Speed of the top plate $d H / d t\left(\mathrm{~m} \mathrm{~s}^{-1}\right)$

$H_{0} \quad$ Initial sample height Fig. 1 (m)

$H^{*} \quad$ Complex function related to sample height $H=$ $\Re\left\{H^{*}\right\}(\mathrm{m})$

$i$ Imaginary unit $\sqrt{-1}(-)$

$m$ Flow consistency index for a power law fluid, Eq. (5) $\left(\mathrm{Pa} \mathrm{s}^{\mathrm{n}}\right)$

$M_{w} \quad$ Weight average molar mass $\left(\mathrm{kg} \mathrm{mol}^{-1}\right)$

$n$ Power law exponent of a power law fluid, Eq. (5) $(-)$

$O \quad$ Order of magnitude indication, Eq. (47) (-)

$p \quad$ Isotropic compressive stress (pressure) $(\mathrm{Pa})$

$p_{\text {atm }} \quad$ Atmospheric pressure $(\mathrm{Pa})$

$\bar{p} \quad$ Complex function related to pressure, Eq. (9) (Pa)

$\hat{p}$ Dimensionless complex function related to pressure, Eq. (15) (-)

$\hat{p}_{0}, \hat{p}_{1} \quad$ Dimensionless complex integration constants for pressure, Eqs. (33) and (60) (-)

$(r, \theta, z) \quad$ Cylindrical coordinates $[(\mathrm{m},-, \mathrm{m})]$

$R$ Sample radius Fig. $1(\mathrm{~m})$

$(\hat{r}, \hat{\theta}, \hat{z}) \quad$ Nondimensional cylindrical coordinates $\left(r / R_{0}, \theta, z / H_{0}\right)[(-,-,-)]$

$R_{0} \quad$ Mean sample radius $\int_{0}^{\infty} R(t) d t / \int_{0}^{\infty} d t(\mathrm{~m})$

Re Complex Reynolds number $i \rho \omega H_{0}^{2} / \eta^{*}(-)$

$t$ Time (s)

$T$ Temperature $(K) /(\mathrm{C})$

$v$ Velocity vector $\partial \boldsymbol{x} / \partial t\left(\mathrm{~m} \mathrm{~s}^{-1}\right)$

$V \quad$ Sample volume $\left(\mathrm{m}^{3}\right)$

$\bar{v}$ Complex function related to velocity vector, Eq. (8) $\left(\mathrm{m} \mathrm{s}^{-1}\right)$

$\hat{\boldsymbol{v}}$ Dimensionless complex function related to velocity, Eqs. (13) and (14) (-)

$v_{s}$ Slip velocity at the wall $v_{r}(z=0)$ or $v_{r}(z=H)$ $\left(\mathrm{m} \mathrm{s}^{-1}\right)$

$\boldsymbol{x}$ Position vector

$\hat{\boldsymbol{x}}$ Nondimensional position vector

$\beta \quad$ Slip coefficient, Eq. (69) $\left(\mathrm{m}^{2} \mathrm{~s} / \mathrm{kg}\right)$

$\gamma \quad$ Shear strain, Eq. (68) (-)

$\dot{\gamma}_{r z} \quad$ Shear rate $\partial v_{r} / \partial z\left(\mathrm{~s}^{-1}\right)$

$\eta \quad$ Newtonian viscosity $\boldsymbol{\sigma} / \dot{\gamma}(\mathrm{Pa} \mathrm{s})$

$\eta^{*} \quad$ Complex viscosity $\left(G^{\prime \prime}-i G^{\prime}\right) / \omega(\mathrm{Pa} \mathrm{s})$

$\Lambda \quad$ Aspect ratio of the sample $H_{0} / R_{0}(-)$

$\rho$ Density of the fluid $\left(\mathrm{kg} \mathrm{m}^{-3}\right)$

$\sigma \quad$ Extra stress tensor $(\mathrm{Pa})$

$\hat{\boldsymbol{\sigma}}$ Dimensionless complex stress tensor, Eqs. (29) and (30) (-)

$\phi_{V} \quad$ Viscous dissipation term, Eq. (40) $\left(\mathrm{s}^{-2}\right)$

$\omega$ Oscillation frequency of the top plate $\left(\mathrm{rad} / \mathrm{s}^{-1}\right)$

\section{References}

[1] Engmann, J., C. Servais, and A. S. Burbidge, "Squeeze flow theory and applications to rheometry: A review," J. Non-Newtonian Fluid Mech. 132, 1-27 (2005).

[2] Standard Test Method for Apparent Viscosity (Flow) of Roofing Bitumens Using Parallel Plate Plastometer, American Society for Testing and Materials, West Conshocken, PA (2014).

[3] Bird, R. B., R. C. Armstrong, and O. Hassager, Dynamics of Polymeric Liquids. Vol. 1: Fluid Mechanics, 2nd ed. (John Wiley, New York, 1987).

[4] Dienes, G. J., and H. F. Klemm, "Theory and applications of parallel plate plastometer," J. Appl. Phys. 17, 458-471 (1946).

[5] Gent, A. N., "Theory of the parallel plate viscometer," Br. J. Appl. Phys. 11, 85-87 (1960).

[6] Gibson, A. G., G. Kotsikos, J. H. Bland, and S. Toll, "Squeeze flow," in Rheological Measurement, 2nd ed. (Chapman and Hall, London, 1998), Chap. 18.

[7] Pham, H., and E. A. Meinecke, "Squeeze film rheology of polymer melts: Determination of the characteristic flow curve," J. Appl. Polym. Sci. 53, 257-264 (1994).

[8] Pham, H., and E. A. Meinecke, "Squeeze film flow processability tester," J. Appl. Polym. Sci. 53, 265-273 (1994).

[9] Shaw, M. T., "Melt characterization of ultra-high molecular weight polyethylene using squeeze flow," Polym Eng. Sci. 17, 266-268 (1977).

[10] Cua, E. C., and M. T. Shaw, "Using creeping squeeze flow to obtain low-frequency linear viscoelastic properties: Low-shear rate measurements on polydimethyloxane," J. Rheol. 46, 817-830 (2002).

[11] Chatraei, S. H., C. W. Macosko, and H. H. Winter, "Lubricated squeeze flow: A new biaxial extensional rheometer," J. Rheol. 25, 433-443 (1981).

[12] Nishioka, A., Y. Takagi, T. Takashashi, Y. Masubuchi, J. Takimoto, and K. Koyama, "Measurement of biaxial elongational viscosity of polymer melts using lubricated squeezing flow method," J. Soc. Mater. Sci. Jpn. 47, 1296-1300 (1998).

[13] Kompani, M., and D. C. Venerus, "Equibiaxial extensional flow of polymer melts via lubricated squeezing flow I. Experimental analysis," Rheol. Acta 39, 444-451 (2000).

[14] Guaderama-Medina, T., T.-Y. Shiu, and D. C. Venerus, "Direct comparison of equibiaxial elongational viscosity measurements and the multiaxiales dehnrheometer," Rheol. Acta 48, 11-17 (2009).

[15] Hachmann, P., and J. Meissner, "Rheometer for equibiaxial and planar elongations of polymer melts," J. Rheol. 47, 989-1010 (2003).

[16] Pipkin, A. C., Lectures on Viscoelasticity Theory (Springer, Berlin, 1972).

[17] Phan-Thien, N., "Small strain oscillatory squeeze film flow of simple fluids," J. Aust. Math. Soc. (B) 22, 22-37 (1980).

[18] Field J. S., M. V. Swain, and N. Phan-Thien, "An experimental investigation of the use of random squeezing to determine the complex modulus of viscoelastic fluids," J. Non-Newtonian Fluid Mech. 65, 177-194 (1996).

[19] Konigsberg, D., T. M. Nicholson, P. J. Halley, and K. H. Ahn, "Technical note: Correcting for shear strain in an oscillatory squeeze flow rheometer," Rheol. Acta 53, 103-107 (2014).

[20] Phan-Tien, N., J. S. Field, and M. V. Swain, "Micro-Fourier rheometer: Inertial effects," Rheol. Acta 35, 410-416 (1996).

[21] Debbaut, B., and K. Thomas, "Simulation and analysis of oscillatory squeeze flow," J. Non-Newtonian Fluid Mech. 124, 77-91 (2004).

[22] Bell, D., D. M. Binding, and K. Walters, "The oscillatory squeeze flow rheometer: Comprehensive theory and a new experimental facility," Rheol. Acta 46, 111-121 (2006). 
[23] Bird, R. B., W. E. Stewart, and E. N. Lightfoot, Transport Phenomena, 2nd ed. (John Wiley, New York, 2007).

[24] Laun, H. M., M. Rady, and O. Hassager, "Analytical solutions for squeeze flow with partial wall slip," J. Non-Newtonian Fluid Mech. 81, 1-15 (1999).

[25] Brindley, G., J. M. Davies, and K. Walters, "Elastico-viscous squeeze films. Part I,” J. Non-Newtonian Fluid Mech. 1, 19-37 (1976).

[26] Whittingstall, P. R., and W. E. Van Arsdale, "A compressional rheometer for viscoelastic fluids," in 70th Annual Meeting, Society of Rheology, Monterrey, CA (1998).

[27] Van Arsdale, W. E., and H. Motivala, "Device for determining viscoelastic properties of liquids and a method for use," U.S. patent 5,253,513 (1993).

[28] Walberer, J. A., and A. J. McHugh, "The linear viscoelastic behavior of highly filled polymethylsiloxine measured in shear and compression,” J. Rheol. 45, 187-201 (2001).

[29] Crassous, J. J., R. Régisser, M. Ballauff, and M. N. Willenbacher, "Characterization of the viscoelastic behavior of complex fluids using the piezoelastic axial vibrator," J. Rheol. 49, 851-863 (2005).

[30] Nelson, B. I., and J. M. Dealy, "Dynamic mechanical analysis using complex waveforms," in Rheological Measurement, 2nd ed. (Chapman and Hall, London, 1998), Chap. 4.

[31] Yaglom, A. M., An Introduction to the Theory of Stationary Random Functions (Prentice Hall, Englewood Cliffs, NJ, 1965).

[32] See, H., P. Jiang, and N. Phan-Tien, "Concentration dependence of the linear viscoelastic properties of particle suspensions," Rheol. Acta 39, 131-137 (2000).

[33] Jiang, P., H. See, M. V. Swain, and N. Phan-Thien, "Using oscillatory flow to measure the viscoelastic properties of dental composite resins during curing," Rheol. Acta 42, 118-122 (2003).

[34] Sakai, S., "Improvements of an oscillatory squeezing flow rheometer for small elasticity measurements of liquids," Rheol. Acta 44, 16-28 (2004).

[35] Konigsberg, D., T. M. Nicholson, P. J. Halley, T. J. Kealy, and P. K. Bhattacharjee, "Online process rheometry using oscillatory squeeze flow," Appl. Rheol. 23(3), 35688-1-35688-10 (2013).

[36] Konigsberg, D., The rheology and fluid mechanics of oscillatory squeeze flow, Doctoral thesis, University of Queensland, St Lucia, Australia, 2014.
[37] Kealy, T., and P. Bhattacharrya, "On-line rheological measurements for industrial process monitoring and control of complex fluids," in Annual European Rheology Conference, 2014.

[38] Bach, A., H. K. Rasmussen, P.-Y. Longin, and O. Hassager, "Growth of non-axisymmetric disturbances of the free surface in the filament stretching rheometer: Experiments and simulation," J. Non-Newtonian Fluid Mech. 108, 163-186 (2002).

[39] Román Marín, J. M., J. K. Huusom, N. J. Alvarez, Q. Huang, H. K. Rasmussen, A. Bach, A. L. Skov, and O. Hassager, "A control scheme for filament stretching rheometers with application to polymer melts," J. Non-Newtonian Fluid Mech. 194, 14-22 (2013).

[40] Wingstrnd, S. L., N. J. Alvarez, and O. Hassager, "Linear viscoelastic characterization from filament stretching rheometry," Annual European Rheology Conference, Nantes, France, 2015.

[41] Hudson, N. E., and T. E. R. Jones, "The A1 project-An overview," J. Non-Newtonian Fluid Mech. 46, 69-88 (1993).

[42] See, H., and P. Nguyen, "Using oscillatory squeeze flow to monitor the change in viscoelastic properties of curing materials," Nihon Reoroji Gakkaishi (J. Soc. Rheol., Japan) 32, 33-39 (2004).

[43] Peng, J., and K.-Q. Zhu, "Oscillatory squeeze flow of electrorheological fluid with transitional electric field," Int. J. Mod. Phys. B 19, 1249-1255 (2005).

[44] See, H., J. S. Field, and B. Pfister, "The response of electrorheological fluid under oscillatory squeeze flow," J. Non-Newtonian Fluid Mech. 84, 149-158 (1999).

[45] Lee, C. Y., and C. Y. Wen, "The oscillatory squeeze flow of electrorheological fluid considering the inertia effect," Smart Mater. Struct. 11, 553-560 (2002).

[46] Kuzhir, P., M. T. López-López, G. Vertelov, Ch. Pradille, and G. Bossis, "Oscillatory squeeze flow of suspensions of magnetic polymerized chains,” J. Phys.: Condens. Matter 20, 204132 (2008).

[47] Kim, J. H., and K. H. Ahn, "A new instrument for dynamical helical squeeze flow which superposes oscillatory shear and squeeze flow," Rev. Sci. Instrum. 83, 085105 (2012).

[48] Kim, J. H., and K. H. Ahn, "Rheological characteristics of poly(ethylene oxide) aqueous solutions under large amplitude oscillatory squeeze flow," Korea-Aust. Rheol. J. 24, 257-266 (2012). 